\title{
Adsorption Behavior and Mechanism of Tripolyphosphate on Synthetic Goethite
}

\author{
Yong Zhong*, Dandan Sheng**, Fazhi Xie****,†, Guolian Li***, Hui Li*****, \\ Xuan Han**, Wenjie Xie**, and Won-Chun Oh ${ }^{*} * * * * * * *$ \\ *Division of Science and Technology, Anhui Jianzhu University, Hefei 230022, China \\ ** School of Materials Science and Chemical Engineering, Anhui Jianzhu University, Hefei 230022, China \\ *** School of Environment and Energy Engineering, Anhui Jianzhu University, Hefei 230022, China \\ ${ }^{* * * *}$ School of Resources and Environment, Anhui Agricultural University, Hefei 230031, China \\ *****Department of Advanced Materials Science \& Engineering, Hanseo University, Seosan 31962, Korea
}

(Received December 5, 2018; Revised January 30, February 7, 2019; Accepted February 11, 2019)

\begin{abstract}
In order to study the transport behavior of tripolyphosphate (TPP) in aqueous solutions, the adsorption process of TPP on synthetic goethite, which exists stably in supergene environment, has been systematically studied. The adsorption properties under different conditions ( $\mathrm{pH}$, electrolyte presence, and temperature) were investigated. The adsorption of TPP in the presence of humic acid (HA)/fulvic acid (FA) has also been discussed in this paper. The results indicated that the adsorption capacity quickly increased within the first hour and equilibrium was reached within $24 \mathrm{~h}$. The adsorption capacity decreased from 1.98 to 0.27 $\mathrm{mg} \cdot \mathrm{g}^{-1}$ upon increasing the $\mathrm{pH}$ from 8.5 to 11.0 , whereas the adsorption of TPP on goethite hardly changed with increasing electrolyte concentration. The results of analysis of the kinetic and isothermal models showed that the adsorption was more in accord with the pseudo second-order equation and Freundlich model. The adsorption capacity decreased obviously regardless of the order of addition of TPP, HA, and goethite. Subsequent addition of FA led to a large increase in the adsorption capacity, which might be attributed to the adsorption ability of FA. According to the predictions of the kinetic and isothermal models and the spectroscopic evidence (X-ray diffraction (XRD), Fourier Transform infrared spectroscopy (FT-IR), and scanning electron microscope (SEM)), the adsorption mechanism may be mainly based on surface complexation and physical adsorption.
\end{abstract}

Key words : Tripolyphosphate, Adsorption, Goethite, Organic matter, Mechanism

\section{Introduction}

A s one of the growth-limiting nutrients, phosphorus is usually regarded as the most dominant factor accelerating water eutrophication. Excessive amounts of phosphorus have been released into aquatic ecosystems, causing undesirable ecological and economic consequences. ${ }^{1)}$ In recent years, increasing attention has focused on agriculture as a pollution source, particularly on phosphorus transfer. ${ }^{2)}$ The cycle of phosphorus transformations between the various components of the ecosystem has a crucial influence on the water and nutrient balances. ${ }^{3)}$ As one of the forms of bioavailable phosphorus that exists in the environment, condensed phosphates have been less investigated. ${ }^{4)}$ Although some of the condensed phosphates are not directly bioavailable, they can be hydrolyzed to orthophosphates in certain

Corresponding author: Fazhi Xie

E-mail : fzxie@mail.ustc.edu.cn, 541274754@qq.com

Tel : +86-0551-63828106 Fax : +86-0551-63828106

"Corresponding author: Won-Chun Oh

E-mail : wc_oh@hanseo.ac.kr

Tel : +82-41-660-1337 Fax : +82-41-660-1337

ORCID

https://orcid.org/0000-0002-0154-7388 conditions, which serve as potential reservoirs for nonpoint phosphorus sources., ${ }^{56)}$ Halliwell et al. reported that the half-life of detergent phosphates (triphosphate) in waste waters was $7.3 \mathrm{~h}$ at $15^{\circ} \mathrm{C}$ and $3.0 \mathrm{~h}$ at $20^{\circ} \mathrm{C}$, with the most likely decomposition mechanism being enzymatic hydrolysis. ${ }^{7)}$ Tripolyphosphate (TPP; $\mathrm{Na}_{5} \mathrm{P}_{3} \mathrm{O}_{10}$, a condensed phosphate) is widely used as an auxiliary washing agent and has the molecular structure - $\left[\mathrm{PO}_{4}\right]-\left[\mathrm{PO}_{3}\right]-\left[\mathrm{PO}_{4}\right]^{8)}$ TPP is also used in the food industry for peptidization, sequestration, cross-linking of starch, and $\mathrm{pH}$ adjustment. ${ }^{9)}$ Previous studies have confirmed that TPP is strongly and rapidly adsorbed to aluminum hydroxide, ternary $\mathrm{CaMgAl}$-layered double hydroxides, and $\mathrm{MgCaFe}-\mathrm{Cl}-\mathrm{LDH}$. Guan et al. ${ }^{8}$ ) suggested that the small crystals formed on the surface of aluminum hydroxide contributed more to the fast adsorption than the surface sites. A synergic enhancement was observed for TPP removal over $\mathrm{MgCaAl}-\mathrm{Cl}-\mathrm{LDH},{ }^{10)}$ since the release of aluminum was in the form of $\mathrm{Al}(\mathrm{OH})^{4-}$ and the transport process of TPP on MgFe-Cl-LDH was mainly surface adsorption and near-edge intercalation. Phosphate adsorption at the solid/solution interface of metal (hydr)oxides, especially iron and aluminum (hydr)oxides, plays an important role in the transport and bioavailability of phosphorus. ${ }^{11,12)}$ Goethite (a-FeOOH) was found to be relevant for organic matter 
adsorption in natural environments. ${ }^{13,14)}$ In addition, natural organic matter (NOM) was also considered to be important for the phosphorus adsorption process. ${ }^{15,16)}$

In the present research, the adsorption of TPP on goethite under different conditions ( $\mathrm{pH}$, electrolyte, time, and temperature) was studied and the effects of NOM (HA, FA) on TPP adsorption were also investigated.

\section{Experimental Procedure}

\subsection{Apparatus and reagents}

Measurements of the $\mathrm{pH}$ were conducted by using a PHS3C pH-meter (Dapu Instrumentation Co., Ltd. Shanghai, China). All the chemicals $\left(\mathrm{Na}_{5} \mathrm{P}_{3} \mathrm{O}_{10}, \mathrm{~K}_{2} \mathrm{~S}_{2} \mathrm{O}_{8}, \mathrm{NaNO}_{3}\right.$, and $\left.\mathrm{Fe}\left(\mathrm{NO}_{3}\right)_{3} \cdot 9 \mathrm{H}_{2} \mathrm{O}\right)$ used in this study were of analytical reagent grade and the glassware used in the experiments were carefully cleaned with double deionized water. The NOM HA and FA were obtained from the soil of Jiufeng Mountain in Beijing city.

\subsection{Goethite synthesis}

Goethite was synthesized by following the procedures of Schwertmann and Cornell. ${ }^{17)}$ In short, $45 \mathrm{~mL}$ of $5 \mathrm{M} \mathrm{KOH}$ was mixed with $25 \mathrm{~mL}$ of $1 \mathrm{M} \mathrm{Fe}\left(\mathrm{NO}_{3}\right)_{3}$ in a vessel, and the solution was quickly diluted with water to $1 \mathrm{~L}$. The above suspension was then held in a closed polyethylene bottle at $70^{\circ} \mathrm{C}$ for $60 \mathrm{~h}$. The resulting suspension was filtered and the goethite slurry washed with deionized water until the solution became $\mathrm{pH}$ neutral.

\subsection{Characterization}

An X-ray diffractometer (Bruker D8 Advance, Germany) equipped with a scintillation detector and a $\mathrm{Cu}$ Ka radiation source was employed to analyze the phase compositions of the samples in the $2 \theta$ range $10^{\circ}$ to $70^{\circ}$. Infrared spectral analysis was performed by using a Nicolet 6700 s spectrometer with $\mathrm{KBr}$ pellet technique with the width ranging from 400 to $4,000 \mathrm{~cm}^{-1}$. The morphology and structure of the samples were studied by scanning electron microscopy (JEOL JSM-6700F). The surface area was determined with an SSA-4200 BET surface area and pore size analyzer to be $28.62 \mathrm{~m}^{2} \cdot \mathrm{g}^{-1}$. All the dried samples including the goethite before and after the adsorption of TPP, HA, FA, TPP+HA, and TPP+FA were milled with mortar and pestle to the required particle size.

\subsection{Analytical methods}

Prior to the determination of the TP, the solution was separated and filtered through a $0.22 \mu \mathrm{m}$ micropore filter membrane to remove the solid particles. The concentration of polyphosphate in the solution was expressed in total phosphorus $\left(\mathrm{C}_{\mathrm{TP}}\right)$. The concentrations of TP were determined by the molybdenum blue method after digestion with $\mathrm{K}_{2} \mathrm{~S}_{2} \mathrm{O}_{8}{ }^{18)}$ Total organic carbon (TOC) refers to the concentration of $\mathrm{NOM}$ (HA, FA) in the solution, which was determined with a TOC analyzer (Liqui TOC II, Elementar).

\subsection{Adsorption experiment}

TPP solutions with $[\mathrm{TP}]=0-30 \mathrm{mg} \cdot \mathrm{L}^{-1}$ were prepared by dissolving $\mathrm{Na}_{5} \mathrm{P}_{3} \mathrm{O}_{10}$ in deionized water. The amount of TPP was converted to [TP] in the following work. The removal of TPP was carried out by adding $0.05 \mathrm{~g}$ of goethite into a 50 $\mathrm{ml}$ solution, which was continued for $24 \mathrm{~h}$. All the experiments were conducted at $293 \mathrm{~K}$, the initial $\mathrm{pH}$ of 8.5 , and the ionic strength of $0.01 \mathrm{M}$ in a water bath that was agitated at $200 \mathrm{rpm}$. The kinetic experiment was performed in solutions with initial $[\mathrm{TP}]=3,5$, and $10 \mathrm{mg} \cdot \mathrm{L}^{-1}$ within $48 \mathrm{~h}$. The isothermal adsorption experiment was carried out for initial $[\mathrm{TP}]=1.5,5,10$, and $15 \mathrm{mg} \cdot \mathrm{L}^{-1}$ at 278,288 , and 293 $\mathrm{K}$, respectively. The addition of HA and FA was carried out at initial $[\mathrm{TP}]=5 \mathrm{mg} \cdot \mathrm{L}^{-1}$. The order of addition is expressed as follows: Goe + TPP, HA refers to the case where Goe and TPP are first added, followed by HA; HA + TPP, Goe refers to the case where HA and TPP are first added, followed by Goe; Goe + HA, TPP refers to the case where Goe and HA are first added, followed by HA; and Goe + TPP + HA refers to the case of simultaneous addition of all the components.

\section{Results and Discussion}

\subsection{Effects of ionic strength on TPP adsorption}

The effect of ionic strength on TPP removal was studied in $\mathrm{NaNO}_{3}$ solution. The experimental data are shown in Fig. 1. It clearly indicates that the adsorption of TPP on goethite obviously increases with increasing initial TPP concentration, which may be attributed to the sufficient number of active sites in goethite. The increase in the ionic strength can induce a less negative zeta potential at the adsorption plane of goethite, which can remarkably increase the TPP adsorption. ${ }^{19)}$

\subsection{Effects of pH on TPP adsorption}

The $\mathrm{pH}$ is one of the important factors affecting the adsorption capacity. Since TPP is easily hydrolyzed in acidic conditions, ${ }^{20)}$ this research studied the adsorption of TPP in

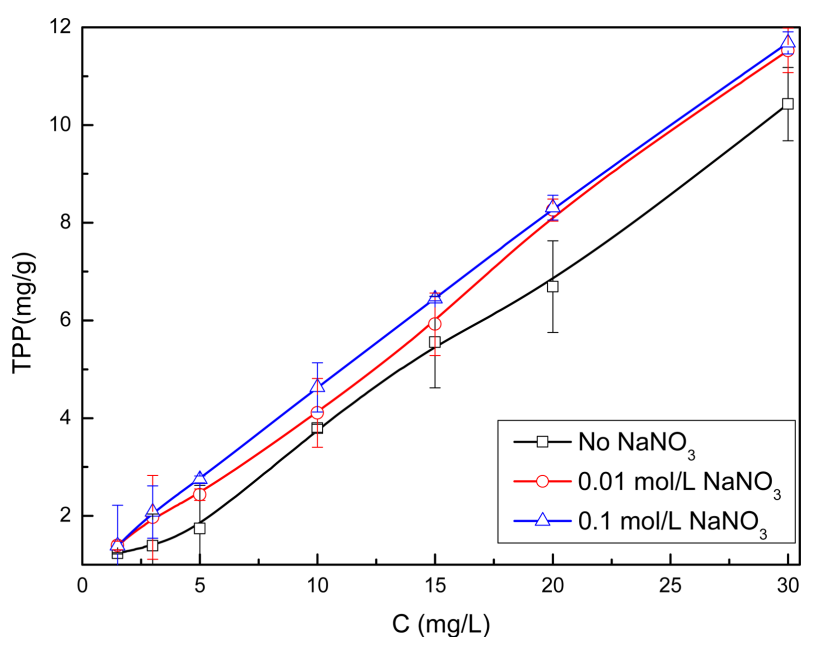

Fig. 1. Influence of electrolyte on adsorption. 


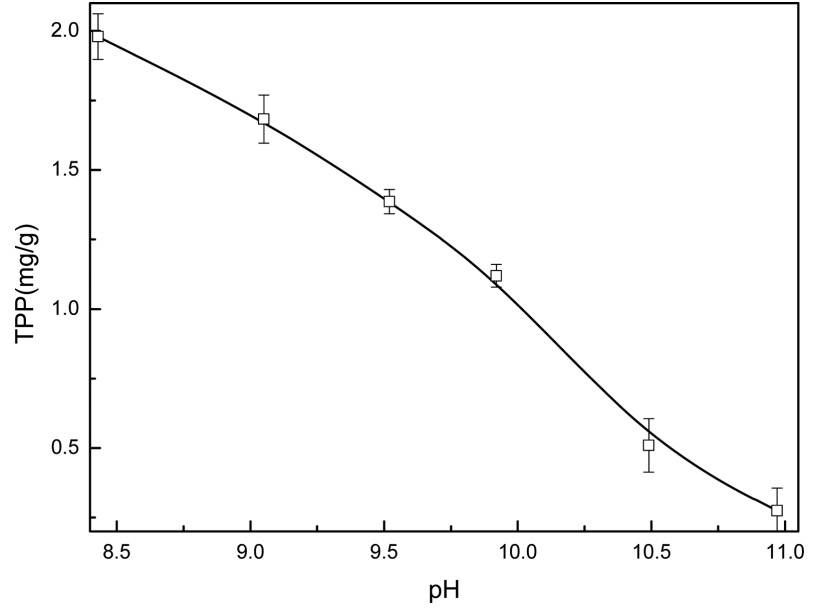

Fig. 2. Influence of $\mathrm{pH}$ on adsorption.

alkaline solutions. The results revealed that the TPP adsorption capacity decreases significantly when the initial $\mathrm{pH}$ increases from 8.5 to 11.0 (Fig. 2). Since the point of zero charge of goethite is about $8.5,{ }^{21)}$ its surface accumulates more negative charge with the increase in the $\mathrm{pH}$ value and produces electrostatic repulsion between the negatively charged sites and the negatively charged TPP ions, thus significantly decreasing the adsorption capacity. ${ }^{22)}$ In addition, the $\mathrm{OH}^{-}$in the solution compete with the TPP ions for the goethite adsorption sites, which may also contribute to the decreased adsorption of TPP.

\subsection{Effects of temperature and adsorption isotherms}

The equilibrium adsorption capacities obtained at different temperatures (278, 288, and $293 \mathrm{~K}$ ) are shown in Fig. 3 and Table 1. The adsorption experiments were carried out at low temperatures to prevent TPP hydrolysis. Langmuir and Freundlich models were used to simulate the adsorption equilibrium data. The linear form of the isotherm model is written $\operatorname{as}^{23)}$

$$
\text { Langmuir: } \frac{C_{e}}{q_{e}}=\frac{1}{b q_{m}}+\frac{C_{e}}{q_{m}}
$$

Freundlich: $\log q_{e}=\log \mathrm{K}_{F}+\frac{1}{n} \log C_{e}$

where $C_{e}$ is the equilibrium adsorption concentration $\left(\mathrm{mg} \cdot \mathrm{L}^{-1}\right), q_{e}$ is the equilibrium adsorption capacity $\left(\mathrm{mg}^{-\mathrm{g}^{-1}}\right)$, $q_{m}$ is the theoretical maximum monolayer adsorption capacity of the adsorbent $\left(\mathrm{mg} \cdot \mathrm{g}^{-1}\right), b$ refers to the adsorption con-

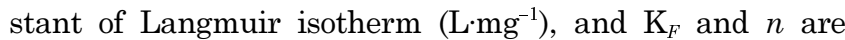

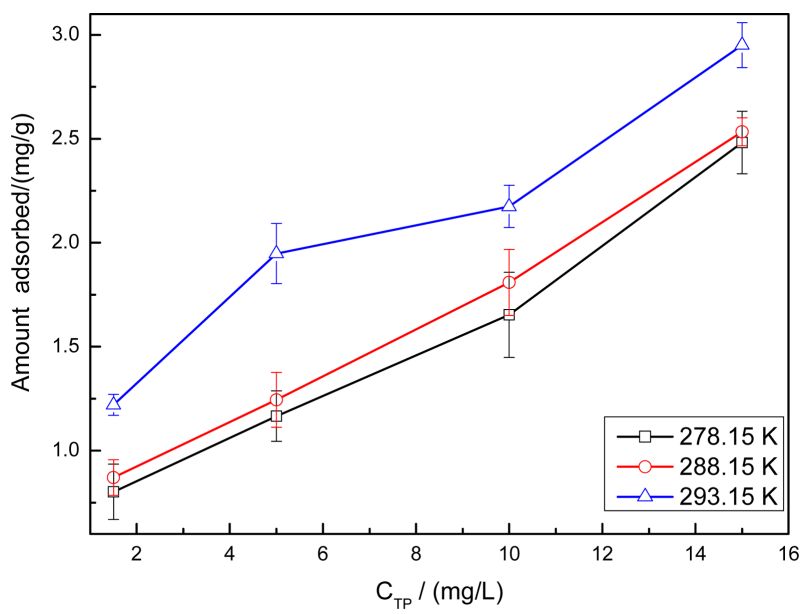

Fig. 3. Influence of temperature on TPP adsorption.

Freundlich constants related to the adsorption capacity and adsorption intensity, respectively. Table 1 summarizes all the adsorption isotherm parameters obtained at different temperatures; the Freundlich isotherm model revealed a higher correlation coefficient, indicating that the adsorption of TPP on goethite was heterogeneous and could be better explained by this model. The value of the constant $\mathrm{K}_{F}$ increased with temperature, which indicated that the goethite adsorption was endothermic. The constant $\mathrm{n}$ varied between 2 and 10 for all the three temperatures, which indicated that adsorption was favored. ${ }^{24)}$ In addition, according to Arrhenius equation (equation 3), the apparent activation energy of the process was $11.27 \mathrm{~kJ} \cdot \mathrm{mol}^{-1}$; the result also showed that the adsorption process included chemical adsorption.

Arrhenius equation: $K=A \cdot e^{-E a / R T}$

In the Arrhenius equation, $K$ is the reaction rate constant $\left(\mathrm{g} \cdot \mathrm{mg}^{-1} \cdot \mathrm{min}^{-1}\right)$, A is the frequency factor, $E_{a}$ is the apparent activation energy $\left(\mathrm{kJ} \cdot \mathrm{mol}^{-1}\right), R$ is the molar gas constant $\left(\mathrm{J} \cdot \mathrm{K}^{-1} \cdot \mathrm{mol}^{-1}\right)$, and $T$ is the reaction temperature $(\mathrm{K})$.

\subsection{TPP adsorption kinetics}

The experimental results of the TPP adsorption kinetics are shown in Fig. 4 and Table 2. The three different adsorption curves suggested that the process occurred faster within the first hour, before becoming slower as the adsorption time increased. Therefore, the adsorption equilibrium time was determined to be less than $24 \mathrm{~h}$. According to the equilibrium adsorption capacity, the pseudo-first-order and pseudo-second-order equations were used to explain the

Table 1. Parameters of the Freundlich and Langmuir Models

\begin{tabular}{ccccccc}
\hline \multirow{2}{*}{$\begin{array}{c}\text { Temperature } \\
(\mathrm{K})\end{array}$} & \multicolumn{3}{c}{ Langmuir constants } & \multicolumn{3}{c}{ Freundlich constants } \\
\cline { 2 - 7 } & $q_{m}\left(\mathrm{mg} \cdot \mathrm{g}^{-1}\right)$ & $b\left(\mathrm{~L} \cdot \mathrm{mg}^{-1}\right)$ & $R^{2}$ & $\mathrm{~K}_{F}$ & $n$ & $R^{2}$ \\
\hline 278 & 2.87 & 0.26 & 0.83 & 0.84 & 2.78 & 0.90 \\
288 & 2.90 & 0.30 & 0.88 & 0.94 & 2.99 & 0.91 \\
293 & 2.98 & 0.79 & 0.94 & 1.57 & 4.74 & 0.94 \\
\hline
\end{tabular}




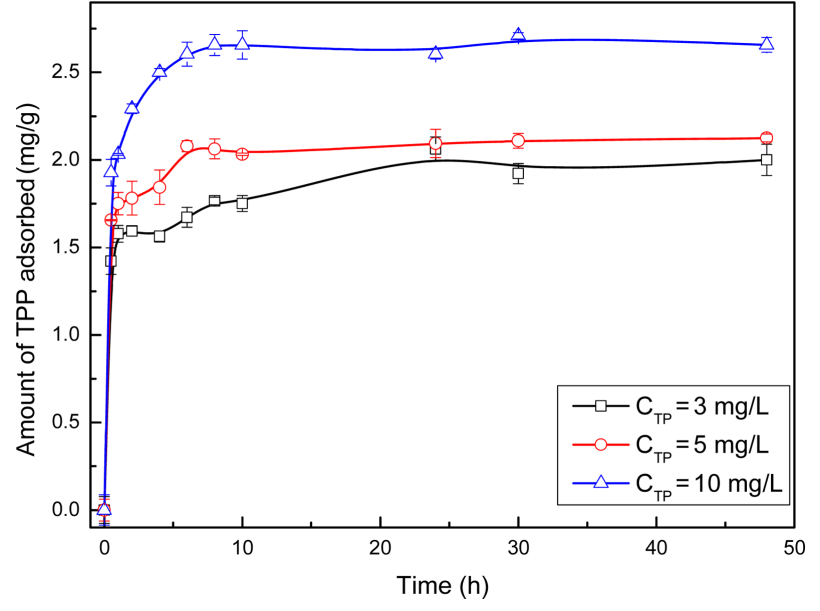

Fig. 4. Influence of time on adsorption.

adsorption mechanism of TPP removal:

Pseudo-first-order rate equation: $\ln \left(q_{e}-q_{t}\right)=\ln q_{e}-k_{1} t$

Pseudo-second-order rate equation: $\frac{t}{q_{t}}=\frac{1}{k_{2} q_{e}^{2}}+\frac{1}{q_{e}} t$

where $q_{t}\left(\mathrm{mg} \cdot \mathrm{g}^{-1}\right)$ is the adsorption capacity at the contact time $t(\mathrm{~h}), q_{e}$ is the equilibrium adsorption capacity $\left(\mathrm{mg} \cdot \mathrm{g}^{-1}\right)$, $k_{1}\left(\mathrm{~min}^{-1}\right)$ is the pseudo-first-order rate constant, and $k_{2}$ $\left(\mathrm{g} \cdot \mathrm{mg}^{-1} \cdot \mathrm{min}^{-1}\right)$ is the pseudo-second-order equilibrium rate constant.

The parameters of the pseudo-first-order and pseudo-second-order models were calculated based on the experimental data and the results are presented in Table 2. It was observed that the correlation coefficients were very close to 1.0 and that the calculated adsorption capacity $q_{e(\mathrm{cal})}$ of the pseudo-second-order model agreed well with the experimental equilibrium adsorption capacity $q_{e(\exp )}$. The pseudo-firstorder equation only described the initial stage (the first 10 min) of the adsorption, whereas the pseudo-second-order equation could well predict the entire adsorption process.

\subsection{Effects of HA and FA}

The TPP adsorption capacities of goethite with or without HA are shown in Fig. 5. The higher the concentration of HA added to the system (increasing from 0 to $100 \mathrm{mg} \cdot \mathrm{L}^{-1}$ ), the lower was the amount of TPP adsorbed (decreased from 2.41 to $1.14 \mathrm{mg} \cdot \mathrm{g}^{-1}$ ), which indicated that both TPP and HA competed for adsorption on the goethite surface. Differences in the addition orders of TPP, HA, and goethite resulted in minimal changes to the goethite adsorption capacity. The

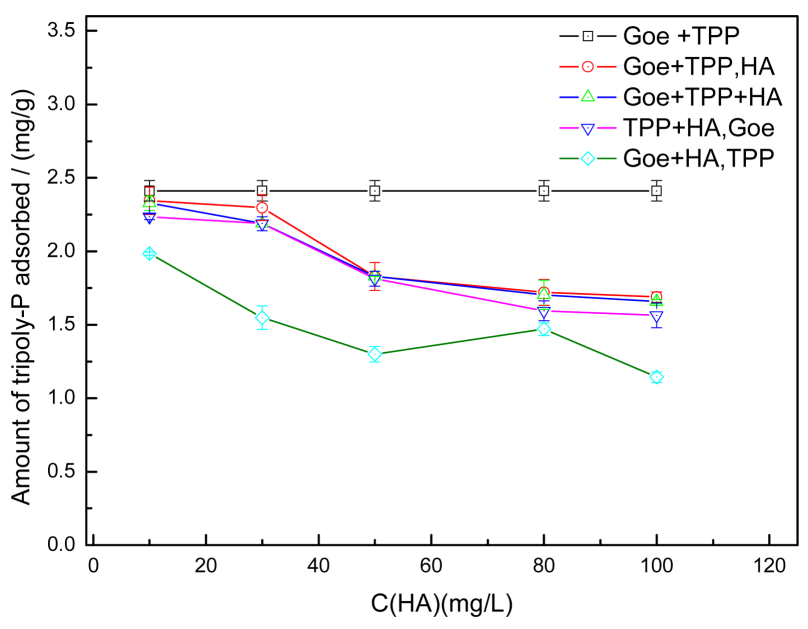

Fig. 5. Influence of HA on adsorption.

adsorption capacity of Goe + TPP, HA > that of Goe + HA, TPP, which indicated that HA could not outdo the TPP adsorbed on the surface of goethite. In addition, HA was strongly bonded to goethite, and was not easily replaced by TPP either.

For a comparison, the effect of FA on TPP adsorption is also presented. The data are displayed in Fig. 6, where the adsorption capacity increased obviously (from 2.41 to 3.49 $\mathrm{mg} \cdot \mathrm{g}^{-1}$ ) after the addition of different amounts of FA (from 0 to $100 \mathrm{mg} \cdot \mathrm{L}^{-1}$ ) into the mixed solution of goethite and TPP; on the other hand, the adsorption capacity showed little change when TPP was added to the mixed solution of goethite and FA. The reason might be that TPP not only

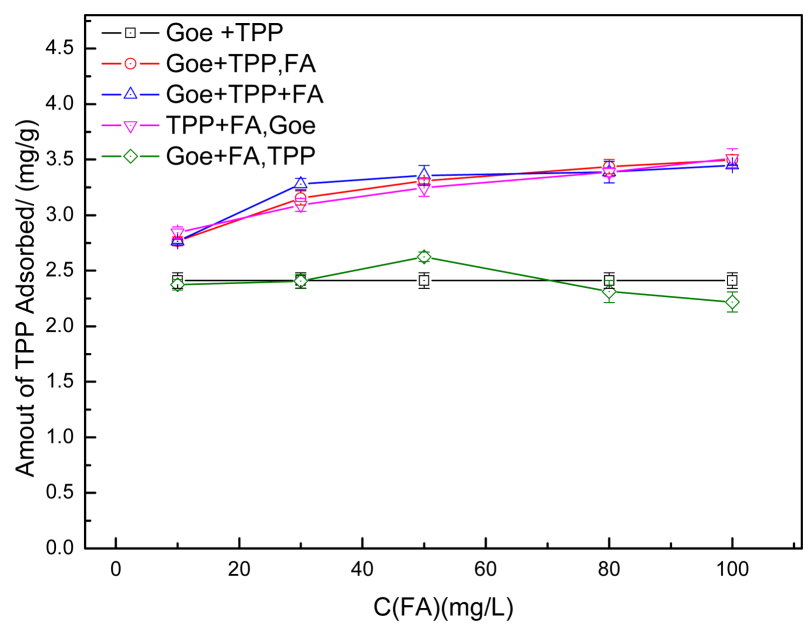

Fig. 6. Influence of FA on adsorption.

Table 2. Parameters of the Kinetic Models

\begin{tabular}{|c|c|c|c|c|c|c|c|}
\hline \multirow{2}{*}{$\mathrm{C}_{\mathrm{TP}}\left(\mathrm{mg} \cdot \mathrm{L}^{-1}\right)$} & \multirow{2}{*}{$\begin{array}{c}q_{e(\exp )} \\
\left(\mathrm{mg} \cdot \mathrm{g}^{-1}\right)\end{array}$} & \multicolumn{3}{|c|}{ Pseudo-first-order equation } & \multicolumn{3}{|c|}{ Pseudo-second-order equation } \\
\hline & & $q_{e(\mathrm{cal})}\left(\mathrm{mg} \cdot \mathrm{g}^{-1}\right)$ & $k_{1}\left(\min ^{-1}\right)$ & $R^{2}$ & $q_{e(\mathrm{cal})}\left(\mathrm{mg} \cdot \mathrm{g}^{-1}\right)$ & $k_{2}\left(\mathrm{~g} \cdot \mathrm{mg}^{-1} \cdot \mathrm{min}^{-1}\right)$ & $R^{2}$ \\
\hline 3 & 2.00 & 0.48 & 0.001 & 0.95 & 2.00 & 0.013 & 0.99 \\
\hline 5 & 2.12 & 0.42 & 0.002 & 0.98 & 2.00 & 0.028 & 0.99 \\
\hline 10 & 2.71 & 0.81 & 0.005 & 0.96 & 2.50 & 0.033 & 0.99 \\
\hline
\end{tabular}




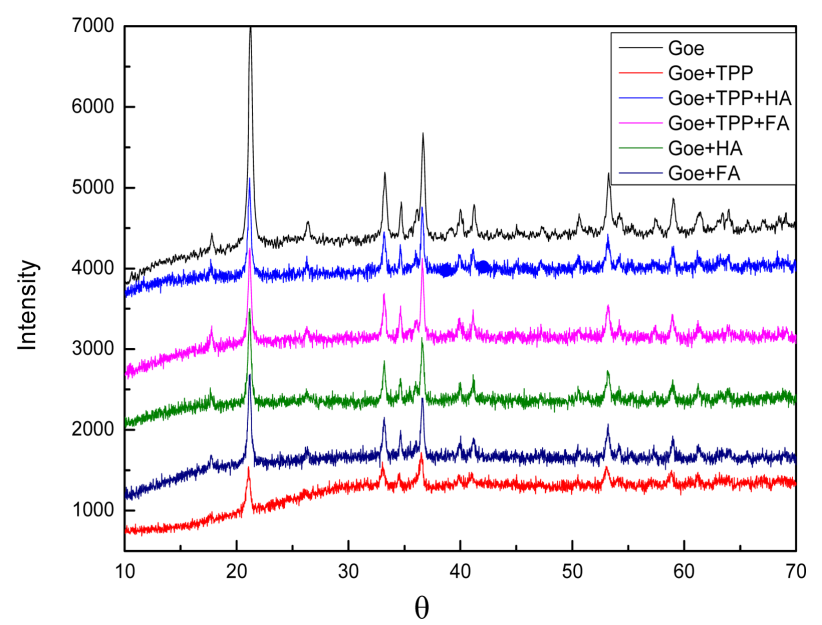

Fig. 7. XRD spectra of unloaded goethite, TPP/HA/FA adsorbed goethite, and goethite with TPP adsorption in the presence of HA/FA.

was bound directly to the sorption sites in goethite but also formed FA-TPP-iron complexes, which then coated on the surface of goethite to increase the sorption capacity of TPP on goethite. ${ }^{25,26)}$

\subsection{Mechanism}

The X-ray diffractograms of goethite before and after the adsorption of TPP are presented in Fig. 7.

The peaks coincided well with those of pure goethite, which revealed that the adsorption did not change the structure of the inner goethite. However, the intensities of those XRD peaks decreased, which was possibly due to a portion of the XRD signal being related to the adsorbed organic matter, which suppressed the signal corresponding to goethite. ${ }^{27)}$

The IR spectra of unloaded and loaded goethite are shown in Fig. 8. Compared to that of unloaded goethite, the IR spectrum of loaded goethite revealed two bands (1,044 and

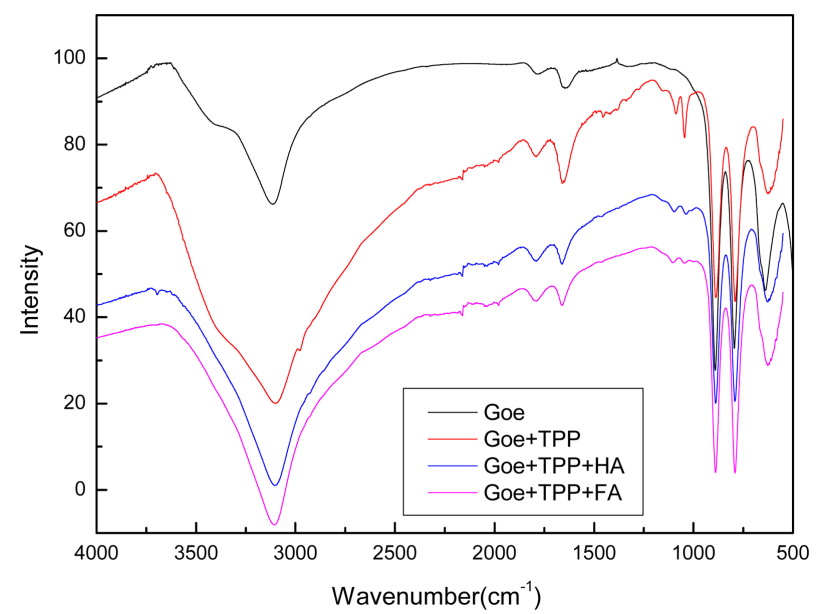

Fig. 8. FT-IR spectra of unloaded and TPP adsorbed goethite and goethite with TPP adsorbed in the presence of HA/FA.

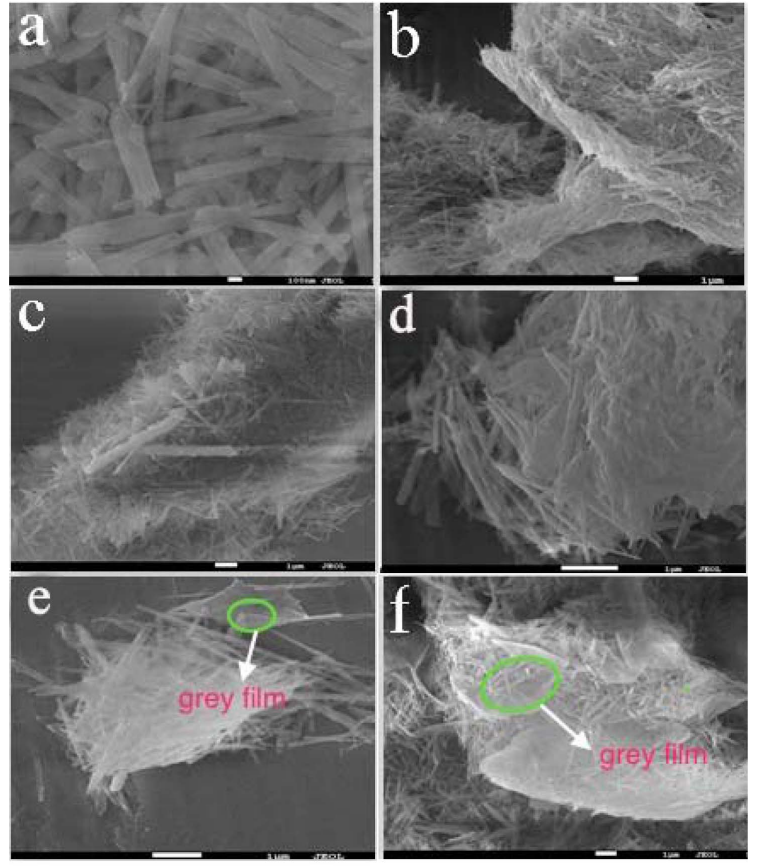

Fig. 9. SEM images of goethite that is unloaded (a), TPP adsorbed (b), and TPP adsorbed in the presence of HA (c)/FA (d), HA (e)/FA (f).

$\left.1,087 \mathrm{~cm}^{-1}\right)$, which were assigned to the overlapping asymmetric $\left(1,087 \mathrm{~cm}^{-1}\right)$ and symmetric $\left(1,044 \mathrm{~cm}^{-1}\right)$ stretchings of the terminal $\mathrm{PO}_{4}{ }^{3-}{ }^{28)}$ The appearance of new peaks and the change in intensity in the FT-IR spectra at different adsorption conditions suggested that the adsorption process may form a new complex.

Figure 9 shows the SEM images of goethite before and after the adsorption of TPP and HA/FA. Goethite before being treated with phosphate (Fig. 9(a)) exhibited long, acicular (needle-like) crystals and a more regular surface. Each goethite particle after adsorption showed aggregation. The samples prepared with TPP and FA illustrated not only the aggregation but also the formation of a light grey film on the surface of goethite, which might be attributed to the reaction of TPP and goethite-FA complexes.

\section{Conclusions}

In the current work, the removal of TPP by using synthetic goethite was investigated systematically. It was found that the adsorption capacity decreased obviously with increasing $\mathrm{pH}$, but changed little with increasing electrolyte concentration. As for the results of the kinetic studies, the absorption capacity quickly increased within $1 \mathrm{~h}$ and equilibrium was reached within $24 \mathrm{~h}$. The adsorption results of this study fitted well with the pseudo-second-order rate equation and Freundlich model. Different orders of addition of TPP, HA, and goethite resulted in different adsorption capacities of goethite: Goe + TPP, HA $\approx$ Goe $+\mathrm{TPP}+\mathrm{HA} \approx$ $\mathrm{HA}+\mathrm{TPP}$, Goe $>$ Goe + HA, TPP. Subsequent addition of FA could increase the TPP absorption capacity of goethite, 
which might be attributed to the adsorption of TPP on FA. Characterization of the materials showed no change in the internal goethite, which revealed that the adsorption mechanism may be mainly based on surface complexation and electrostatic adsorption.

\section{Acknowledgments}

This research was financially supported by the National Science Foundation of China (21777001), Foundation of Anhui Provincial Department of Education (KJ2018ZD049), and Natural Science Foundation of Anhui Province (1608085MB43).

Conflicts of Interest: The authors declare no conflict of interest.

\section{REFERENCES}

1. G. K. Nürnberg, L. A. Molot, E. O'Connor, H. Jarjanazi, J. Winter, and J. Young, "Evidence for Internal Phosphorus Loading, Hypoxia and Effects on Phytoplankton in Partially Polymictic Lake Simcoe, Ontario," J. Great Lakes Res., 39 [2] 259-70 (2013).

2. Z. He, J. G. Hiscock, A. Merlin, L. Hornung, Y. Liu, and J. Zhang, "Phosphorus Budget and Land Use Relationships for the Lake Okeechobee Watershed, Florida," Ecol. Eng., 64 325-36 (2014).

3. Y. Gao, Z. Hao, T. Yang, N. P. He, X. F. Wen, and G. R. $\mathrm{Yu}$, "Effects of Atmospheric Reactive Phosphorus Deposition on Phosphorus Transport in a Subtropical Watershed: A Chinese Case Study," Environ. Pollut., 226 69-78 (2017).

4. X. H. Guan, G. H. Chen, and C. Shang, "Adsorption Behavior of Condensed Phosphate on Aluminum Hydroxide," J. Environ. Sci., 19 [3] 312-18 (2007).

5. A. O. Babatunde, Y. Q. Zhao, Y. Yang, and P. Kearney, "Reuse of Dewatered Aluminium-Coagulated Water Treatment Residual to Immobilize Phosphorus: Batch and Column Trials Using a Condensed Phosphate," Chem. Eng. J., 136 [2-3] 108-15 (2008).

6. J. G. Hamilton, D. Hilger, and D. Peak, "Mechanisms of Tripolyphosphate Adsorption and Hydrolysis on Goethite," J. Colloid Interface Sci., 491 190-98 (2017).

7. D. J. Halliwell, I. D. Mckelvie, and B. T. Hart, "Hydrolysis of Triphosphate from Detergents in a Rural Waste Water System," Water Res., 35 [2] 448-54 (2001).

8. X. Guan, Adsorption of Phosphates and Organic Acids on Aluminum Hydroxide in Aquatic Environment: Mechanisms and Interactions, pp. 48-55, in Ph.D. Thesis, The Hong Kong University of Science Technology, Hong Kong, 2005.

9. J. Xie, P. Yuan, and R. Jenkins, "Impact of Encapsulation of Sodium Tripolyphosphate on Cook Yield and Oxidative Quality of Beef Patties," Meat Sci., 101 116-16 (2015).

10. J. Zhou, Z. P. Xu, S. Qiao, Q. Liu, Y. Xu, and G. Qin, "Enhanced Removal of Triphosphate by MgCaFe-Cl-LDH: Synergism of Precipitation with Intercalation and Surface Uptake,” J. Hazard. Mater., 189 [1-2] 586-94 (2011).

11. W. Li, L. Wang, F. Liu, X. Liang, X. Feng, W. Tan, L. Zheng, and $\mathrm{H}$. Yin, "Effects of $\mathrm{Al}^{3+}$ Doping on the Structure and
Properties of Goethite and its Adsorption Behavior towards Phosphate," J. Environ. Sci., 45 18-27 (2016).

12. D. Mitrogiannis, M. Psychoyou, I. Baziotis, V. J. Inglezakis, N. Koukouzas, N. Tsoukalas, D. Palles, E. Kamitsos, G. Oikonomou, and G. Markou, "Removal of Phosphate from Aqueous Solutions by Adsorption onto $\mathrm{Ca}(\mathrm{OH})_{2}$, Treated Natural Clinoptilolite," Chem. Eng. J., 320 510-22 (2017).

13. J. M. Arroyave, C. C. Waiman, G. P. Zanini, and M. J. Avena, "Effect of Humic Acid on the Adsorption/Desorption Behavior of Glyphosate on Goethite. Isotherms and Kinetics," Chemosphere, 145 34-41 (2016).

14. Y. Zhang, L. Han, and K. Yu, "The Role of Dissolved Organic Matter in the Competitive Adsorption to Goethite, during Arsenic Mobilization," Procedia Earth Planet. Sci., 17424 27 (2017).

15. H. R. Sindelar, M. T. Brown, and T. H. Boyer, "Effects of Natural Organic Matter on Calcium and Phosphorus CoPrecipitation," Chemosphere, 138 218-24 (2015).

16. Y. Zhu, F. Wu, Z. He, J. P. Giesy, W. Feng, Y. Mu, C. Feng, X. Zhao, H. Liao, and Z. Tang, "Influence of Natural Organic Matter on the Bioavailability and Preservation of Organic Phosphorus in Lake Sediments," Chem. Geol., 397 51-60 (2015).

17. U. Schwertmann and R. M. Cornell, Iron Oxides in the Laboratory: Preparation and Characterization; pp. 136-48, Wiley Online Library, Weinheim, 1991.

18. A. E. Greenberg, L. S. Clesceri, and A. D. Eaton, Standard methods for the Examination of Water and Wastewater; pp. 368-75, American Public Health Association, New Jersey, 1992.

19. X. U. Renkou, W. Yong, and D. Tiwari, "Effect of Ionic Strength on Adsorption of As(III) and As(V) on Variable Charge Soils," J. Environ. Sci., 21 [7] 927-32 (2009).

20. X. H. Guan, Q. Liu, G. H. Chen, and C. Shang "Surface Complexation of Condensed Phosphate to Aluminum Hydroxide: an ATR-FTIR Spectroscopic Investigation," J. Colloid Interface Sci., 289 [2] 319-27 (2005).

21. Y. Gao and A. Mucci, "Acid Base Reactions, Phosphate and Arsenate Complexation, and their Competitive Adsorption at the Surface of Goethite in $0.7 \mathrm{M} \mathrm{NaCl}$ Solution," Geochim. Cosmochim. Acta, 65 [4] 2361-78 (2001).

22. Y. Zhao, F. Liu, and X. Qin, "Adsorption of Diclofenac onto Goethite: Adsorption Kinetics and Effects of $\mathrm{pH}$," Chemosphere, 180 373-78 (2017).

23. S. Chen, Q. Yue, B. Gao, and X. Xu, "Equilibrium and Kinetic Adsorption Study of the Adsorptive Removal of Cr(VI) Using Modified Wheat Residue," J. Colloid Interface Sci., 349 [1] 256-64 (2010).

24. B. Noroozi, G. A. Sorial, H. Bahrami, and M. Arami, "Equilibrium and Kinetic Adsorption Study of a Cationic Dye by a Natural Adsorbent-Silkworm Pupa," J. Hazard. Mater., 139 [1-2] 167-74 (2007).

25. J. Gerke and R. Hermann, "Adsorption of Orthophosphate to Humic-Fe-Complexes and to Amorphous Fe-Oxide," $J$. Plant Nutr. Soil Sci., 155 [3] 233-36 (2010).

26. C. N. Guppy, N. W. Menzies, P. W. Moody, and F. P. C. Blamey, "Competitive Sorption Reactions between Phosphorus and Organic Matter in Soil: A Review," Aust. J. Soil Res., 43 [2] 189-202 (2005). 
27. D. Fang, H. J. Tian, X. Ye, C. L. He, Y. M. Dan, and S. Y. Wei, "Adsorption Properties of Fluorine onto Fulvic Acid-

Bentonite Complex," Environ. Sci., 37 [3] 1023-31 (2016).

28. J. Z. Zhou, Z. P. Xu, S. Qiao, J. Liu, Q. Liu, Y. Xu, J. Zhang, and G. Qian, "Triphosphate Removal Processes over Ternary CaMgAl-Layered Double Hydroxides," Appl. Clay Sci., 54 [3-4] 196-201 (2011). 